\title{
Recanalization for Tight Bile Duct-Jejunum Anastomosis Stricture Using Peroral Transliminal Cholangioscopy (with Video)
}

\author{
Takeshi Ogura Akira Miyano Nobu Nishioka Kazuhide Higuchi \\ 2nd Department of Internal Medicine, Osaka Medical College, Osaka, Japan
}

\section{Keywords}

Endoscopic ultrasound - Endoscopic ultrasound-guided hepaticogastrostomy · Cholangioscopy Biliary stricture

\begin{abstract}
Recently, due to improvement of imaging modality, malignant tumor such as pancreatic or bile duct cancer can be detected at earlier stage. Therefore, the frequency of surgical treatment may be increasing. According to this background, benign biliary stricture in anastomosis site may also be increasing. This complication can lead to repeated cholangitis, obstructive jaundice, or liver abscess. Traditionally, interventional radiology such as percutaneous transhepatic biliary drainage (PTCD) or surgical re-anastomosis may be the first choice of treatment for benign biliary stricture in anastomosis site. Recently, double balloon enteroscopy-assisted endoscopic retrograde cholangiopancreatography (DB-ERCP) has emerged. This relatively novel procedure has less invasiveness compared with PTCD or surgical re-anastomosis. However, DB-ERCP has also several disadvantages such as long procedure time or low technical success rate if DB-ERCP is performed by non-expert hands. On the other hand, endoscopic ultrasound-guided biliary drainage has been devel-
\end{abstract}

oped as alternative biliary drainage technique. More recently, novel transluminal approach for biliary tract through endoscopic ultrasound-guided transluminal drainage route has been reported using digital single-operator cholangioscope. We herein report technical tips for peroral transluminal cholangioscopy using digital single-operator cholangioscope, and successfully performed recanalization for tight stricture of bile duct-jejunum anastomosis.

(C) 2018 S. Karger AG, Basel

\section{Introduction}

Endoscopic ultrasound-guided biliary drainage has been developed as alternative biliary drainage technique [1-3]. Recently, novel transluminal approach for biliary tract through endoscopic ultrasound-guided transluminal drainage route has been reported using digital single-operator cholangioscope [4]. We herein report technical tips for peroral transluminal cholangioscopy using digital single-operator cholangioscope, and successfully performed recanalization for tight stricture of bile ductjejunum anastomosis. Patient consent has been obtained.

\section{KARGER}

(C) 2018 S. Karger AG, Basel

E-Mail karger@karger.com

www.karger.com/dd
Takeshi Ogura

2nd Department of Internal Medicine

Osaka Medical College

2-7 Daigakuchou, Takatsukishi, Osaka 569-8686 (Japan)

E-Mail oguratakeshi0411@yahoo.co.jp 
Fig. 1. a Computed tomography shows bile duct dilation, and stricture of bile-jejunum anastomosis is suspected. b Tight bile-jejunum anastomosis stricture is seen on endoscopic ultrasound-guided cholangiography.
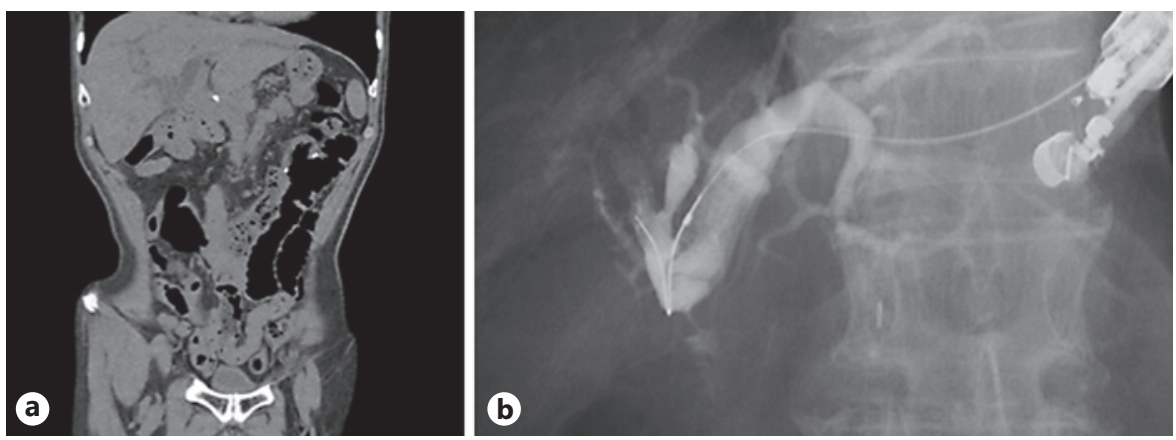
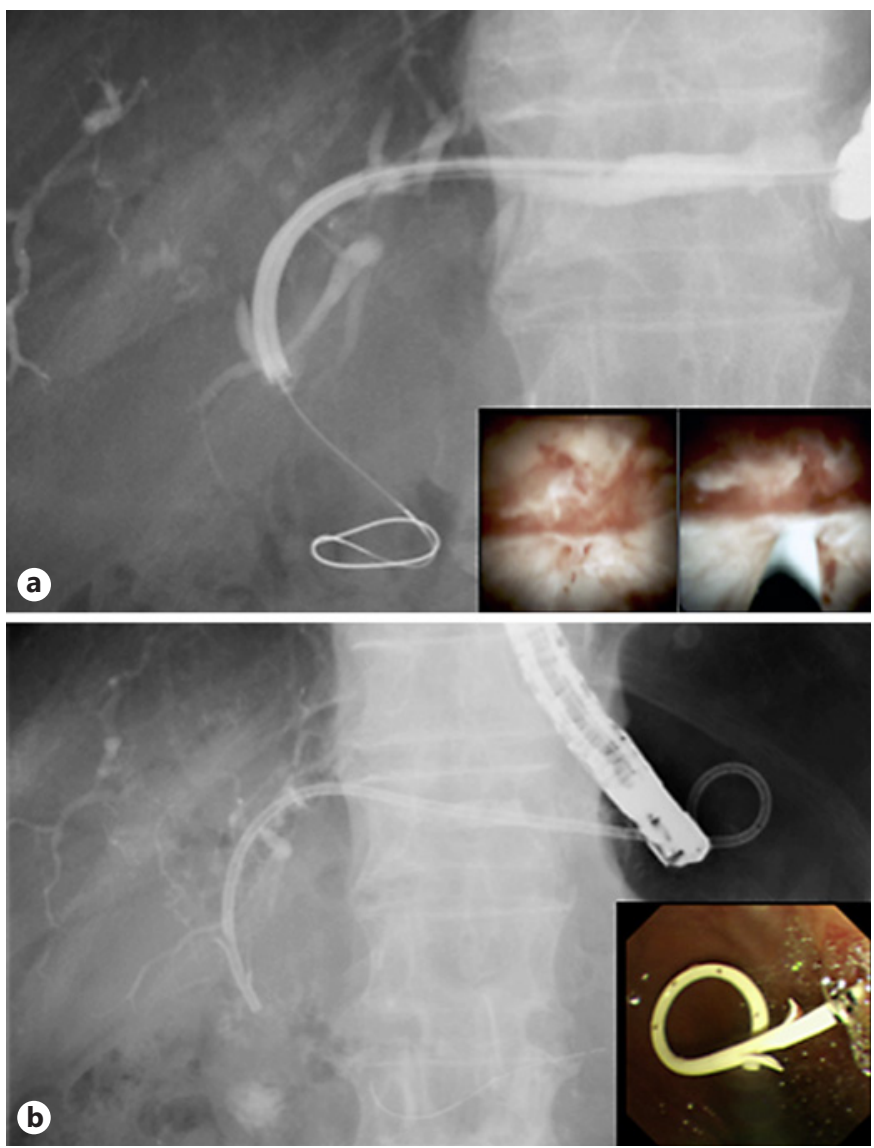

Fig. 2. a The guidewire is successfully inserted into the intestine under transluminal cholangioscopy. b After metal stent removal, stent exchanging is performed from the metal stent to the plastic stent.

\section{Technical Tips}

The presented case was an 82-year-old man, and he was admitted due to obstructive jaundice without tumor maker levels elevation. He underwent pancreaticoduodenectomy due to bile duct cancer 2 years ago. On CT, the intrahepatic bile duct was dilated (Fig. 1a). Echoendoscope (UCT260; Olympus Optical,
Tokyo, Japan), which was connected to an ultrasound device (SSD5500; Aloka, Tokyo, Japan), was inserted into the stomach. The intrahepatic bile duct was punctured using 19-G fine needle aspiration (FNA) needle (Sono Tip Pro Control; Medi-Globe $\mathrm{GmbH}$, Rohrdorf, Germany). After bile juice was aspirated, the contrast medium was injected. Tight bile duct stricture was seen in bile duct-jejunum anastomosis (Fig. 1b). The guidewire could not be advanced into the intestine because of being able to fit the axis; therefore, only fully covered metal stent placement was performed (10 cm length, $10 \mathrm{~mm}$ diameter, Niti-S Biliary Covered Stent; TaeWoong Medical, Seoul, Korea). After 1 week, cholangioscope (SPY DS; Boston Scientific) was inserted into the biliary tract through the fistula. On cholangioscopic findings, almost complete biliary obstruction was seen without any finding suggesting tumor presence. The 0.025 -inch guidewire (VisiGlide; Olympus Medical Systems, Tokyo, Japan) was inserted into the stricture site under direct visualization (Fig. 2a). SPY DS has four-way angle operation; therefore, the recanalization for stricture site was successfully performed. Next, balloon dilation (REN biliary dilation catheter; KANEKA, Osaka, Japan) for the stricture site was performed. Finally, stent placement (Type IT: $7 \mathrm{Fr}$ $\times 12 \mathrm{~cm}$, Gadelius Medical Co., Ltd., Tokyo, Japan) was performed from the intestine to the stomach across the stricture site without any adverse events (Fig. 2b). Result of biopsy specimens was benign, and clinical course was also compatible for benign disease.

\section{Discussion}

Recently, due to improvement of imaging modality, malignant tumor such as pancreatic or bile duct cancer can be detected at earlier stage. Therefore, the frequency of surgical treatment may be increasing. According to this background, benign biliary stricture in anastomosis site may be also increasing. This complication can lead to repeated cholangitis, obstructive jaundice, or liver abscess. Traditionally, interventional radiology such as percutaneous transhepatic biliary drainage (PTCD) or surgical re-anastomosis may be the first choice of treatment of this complication. The technical success rate and complication rates of PTCD for biliary stricture 




Video 1. The intrahepatic bile duct is punctured using 19-G FNA needle, and the contrast medium is injected. The tight stricture of bile-jejunum anastomosis is seen, and the guidewire insertion failed. Endoscopic ultrasound-guided heapticogastrostomy using covered metal stent is performed. Next, SPY DS is inserted into the biliary tract through the HGS stent. Although tumor is not seen in the stricture site, tight stricture is seen. Therefore, the guidewire is inserted under SPY DS guidance and successfully inserted into the intestine through the stricture site.

in anastomosis site were each $55-95 \%$ and $11-35 \%$, and surgical re-anastomosis were each $71-91 \%$ and $20-33 \%$, according to previous reports [5-8]. These results may not be sufficient in clinical practice, and these procedures have several disadvantages such as cosmetic problem or invasiveness. Recently, double balloon enteroscopy-assisted endoscopic retrograde cholangiopancreatography (DB-ERCP) has emerged. This relatively novel procedure has less invasiveness compared with PTCD or surgical re-anastomosis. However, DB-ERCP has also several disadvantages such as long procedure time or low technical success rate if DB-ERCP is performed by non-expert hands $[9,10]$. Compared with double balloon enteroscopic approach, our technique has several advantages. First, procedure time to obtain biliary drainage may be shorter. Second, technical success may be higher. If double balloon enteroscopy can be deeply advanced into the intestine, it may be difficult to detect the anastomosis site. Third, re-intervention may be complicated and need long procedure time because enteroscopy must be advanced into the site of anastomosis site, again. Compared with percutaneous transheshepatic approach, our technique has also several advantages. First, because of internal drainage, patient's quality of life may be kept compared with external drainage. In addition, cosmetic problem may also be superior. Second, PTLC can be performed without stepup fistula dilation process; therefore, the length of hospitalization stay may be shorter. Third, because of direct visualization of anastomosis site, guidewire insertion can be certainly performed. However, this technique is complicated; therefore, bile leak may be more frequent. Therefore, we firstly performed endoscopic ultrasoundguided hepaticogastrostomy (EUS-HGS) using covered metal stent to prevent bile leak, although the cost-effectiveness compared with DB-ERCP or PTCD should be evaluated in a further study. Finally, strategies for benign biliary stricture as presented with the case in our hospital are as follows: EUS-HGS was performed using covered metal stent. After fistula was created, covered metal stent was removed, and we performed stent placement using plastic stent across the stricture site. After several months, we removed this plastic stent and evaluated stricture site. If stricture was not seen, stent placement was not performed. If stricture was still present, we performed stent placement again. Long-term follow-up is needed to determine whether this strategy is suitable for benign biliary stricture, which is a contraindication for ERCP.

In conclusion, our technique may have an impact for benign biliary stricture on anastomosis site; however, more additional cases are needed to evaluate whether this technique can be safely performed.

\section{Disclosure Statement}

The authors declare that there are no conflicts of interest.

\section{Author Contributions}

Takeshi Ogura wrote a paper. Takeshi Ogura, Akira Miyano, Nobu Nishioka, and Kazuhide Higuchi played roles of interpretation of data for the work, revising it critically for important intellectual content, gave final approval of the version to be published, and agreed to be accountable for all aspects of the work in ensuring that questions related to the accuracy or integrity of any part of the work are appropriately investigated and resolved. 


\section{References}

1 Itoi T, Isayama H, Sofuni A, Itokawa F, Kurihara $\mathrm{T}$, Tsuchiya $\mathrm{T}$, et al: Stent selection and tips on placement technique of EUS-guided biliary drainage: transduodenal and transgastric stenting. J Hepatobiliary Pancreat Sci 2011;18:664-672.

2 Wang K, Zhu J, Xing L, Wang Y, Jin Z, Li Z: Assessment of efficacy and safety of EUSguided biliary drainage: a systematic review. Gastrointest Endosc 2016;83:1218-1227.

3 Dhir V, Itoi T, Khashab MA, Park DH, Yuen Bun Teoh A, Attam R, et al: Multicenter comparative evaluation of endoscopic placement of expandable metal stents for malignant distal common bile duct obstruction by ERCP or EUS-guided approach. Gastrointest Endosc 2015;81:913-923.
4 Ogura T, Takagi W, Kurisu Higuchi K: Technical tips for peroral transluminal cholangioscopy using novel single-operator cholangioscope (with videos). J Hepatobiliary Pancreat Sci 2016;23:E25-E29.

5 Schumacher B, Othman T, Jansen M, Preiss C, Neuhaus H: Long-term follow up of percutaneous transhepatic therapy (PTT) in patients with definite benign anastomotic stricture after hepaticojejunostomy. Endoscopy 2001;33:409-415.

6 Fontein D, Gibson R, Collier N, Tse GT, Wang LL, Speer TG, et al: Two decades of percutaneous transjejunal biliary intervention for benign biliary disease: a review of the intervention nature and complications. Insights Imaging 2011;2:557-565.

7 Pellegrini CA, Thomas MJ, Way LW: Recurrent biliary stricture. Patterns of recurrence and outcome of surgical therapy. Am J Surg 1984; 147:175-180.
8 Lillemoe K, Melton G, Cameron J, Pitt HA, Campbell KA, Talamini MA, et al: Postoperative bile duct strictures: management and outcome in the 1990s. Ann Surg 2000;232:430431.

9 De Koning M, Morreels TG: Comparison of double-balloon and single-balloon enteroscope for therapeutic endoscopic retrograde cholangiography after Roux-en-Y bowel surgery. BMC Gastroenterol 2016;22:98.

10 Shah RJ, Smolkin M, Yen R, Ross A, Kozarek RA, Howell DA, et al: A multicenter, U.S. experience of single-balloon, double-balloon, and rotational overtube-assisted enteroscopy ERCP in patients with surgically altered pancreaticobiliary anatomy (with video). Gastrointest Endosc 2013;77:593-600. 